Pacific

Journal of

Mathematics

LIPSCHITZ SOLUTIONS TO THE ISOMETRY RELATION FOR PAIRS OF RIEMANNIAN METRICS

Giuseppina D’ Ambra AND MAhuYa DatTA

Volume $237 \quad$ No. 2

October 2008 


\title{
LIPSCHITZ SOLUTIONS TO THE ISOMETRY RELATION FOR PAIRS OF RIEMANNIAN METRICS
}

\author{
GiUSEPPINA D’AmBRA AND MAHUYA DATTA
}

\begin{abstract}
Let $M$ be a smooth manifold of dimension $n$ with two Riemannian metrics $g_{1}, g_{2}$ which are related by $a^{2} g_{1}<g_{2}<b^{2} g_{1}$. Let $\mathbb{R}^{q}$ be the Euclidean space with two Euclidean metrics $h_{1}, h_{2}$ such that $h_{1}-h_{2}$ has distinct eigenvalues. Further, suppose that $c^{2} h_{1}-h_{2}$ is nondegenerate for each $c \in(a, b)$, and $r_{ \pm}\left(a^{2} h_{1}-h_{2}\right) \geq 2 n$, where $r_{+}$and $r_{-}$denote respectively the positive and the negative ranks of an indefinite metric. Under these conditions we show that there exists an almost everywhere differentiable (Lipschitz) map $f: M \longrightarrow \mathbb{R}^{q}$ satisfying $\left(d f_{x}\right)^{*} h_{i}=g_{i}$ for $i=1,2$ for almost all $x \in M$.
\end{abstract}

\section{Introduction}

It is a classical result due to Nash and Kuiper that a Riemannian manifold $(M, g)$ admitting a $C^{\infty}$-immersion in $\mathbb{R}^{q}$ also admits a $C^{1}$-immersion $f: M \rightarrow \mathbb{R}^{q}$ such that $f^{*} h=g$ provided $q>n$, where $h$ is the canonical metric on $\mathbb{R}^{q}$. Gromov generalised this result via the method of convex integration by showing that if there exists a strictly short immersion of $(M, g)$ into another Riemannian manifold $(N, h)$ then there exists an isometric $C^{1}$-immersion $f: M \rightarrow N$, when $\operatorname{dim} N>\operatorname{dim} M$. He further proved that in the equidimensional case, there are almost everywhere differentiable (Lipschitz) maps whose derivatives $d f$ are isometric almost everywhere on $M$. By an abuse of language, such maps will be referred as the Lipshcitz isometric maps; classically, the Lipschitz maps which preserve the lengths of all rectifiable curves relative to the given metrics are referred as isometric maps. Our notion of Lipschitz isometric maps satisfy a much weaker condition; in fact, such an $f$ may collapse a submanifold of positive codimension in $M$ to a single point.

In this paper we generalise the above mentioned result of Gromov when both the manifolds $M$ and $N$ come with a pair of Riemannian metrics.

MSC2000: 26A16, 58J52.

Keywords: Lipschitz map, isometric immersion, convex integration.

This work is partially done during the visits by the second author to the International Centre for Theoretical Physics as a regular associate. 
Let $M$ be a smooth manifold of dimension $n$. Let $\mathbb{R}^{q}$ be the $q$-dimensional Euclidean space with two Euclidean metrics $h_{1}$ and $h_{2}$ which satisfy the following conditions: There exist two numbers $0<a<b$ such that

(1) $c^{2} h_{1}-h_{2}$ is a nondegenerate indefinite form for each real number $c$ lying in $[a, b]$;

(2) $r_{+}\left(a^{2} h_{1}-h_{2}\right) \geq 2 n$ and $r_{-}\left(b^{2} h_{1}-h_{2}\right) \geq 2 n$, where $r_{+}$and $r_{-}$denote respectively the positive and the negative ranks of an indefinite metric; and

(3) if $A: \mathbb{R}^{q} \rightarrow \mathbb{R}^{q}$ is the unique linear isomorphism given by $h_{2}(v, w)=$ $h_{1}(A v, w)$ for all $v, w \in \mathbb{R}^{q}$, then $A$ has distinct eigenvalues.

Theorem 1.1. Let $g_{1}, g_{2}$ be two Riemannian metrics which are related by $a^{2} g_{1}<$ $g_{2}<b^{2} g_{1}$. Then under assumptions (1)-(3) mentioned above, there exists an almost everywhere differentiable (Lipschitz) map $f: M \rightarrow \mathbb{R}^{q}$ satisfying $\left(d f_{x}\right)^{*} h_{i}=g_{i}$ for $i=1,2$ for almost all $x \in M$. Moreover, such maps are $C^{0}$-dense in the space of strictly $\left(g_{1}, g_{2}\right)$-short maps (see Definition 5.1).

We further observe that if $M$ is a one-dimensional manifold, then (under the same hypothesis) there exists a $C^{1}$-map $f: M \rightarrow \mathbb{R}^{q}$ such that $f^{*} h_{i}=g_{i}$ for $i=1,2$.

The maps $f$ obtained in Theorem 1.1 will be referred as Lipschitz isometric maps for pairs of metrics. If $\mathbb{R}^{q}$ is replaced by a general manifold $N$ in Theorem 1.1 we may have to presuppose the existence of strictly $\left(g_{1}, g_{2}\right)$-short maps in order to conclude the existence of Lipschitz isometric maps [Gromov 1986, 2.4 .9 (A)]. It may be observed that $\left(g_{1}, g_{2}\right)$-short maps always exist for $N=\mathbb{R}^{q}$ (see Proposition 5.2).

In our earlier paper [D'Ambra and Datta 2002] we proved the existence of isometric $C^{1}$-immersions $M \rightarrow \mathbb{R}^{q}$ for pairs of Riemannian metrics when

$$
r_{ \pm}\left(c^{2} h_{1}-h_{2}\right) \geq 3 n+2
$$

for all $c \in[a, b]$, generalizing the Nash-Kuiper $C^{1}$-immersion theorem. The proof was based on Nash's technique for obtaining isometric $C^{1}$-immersions.

In the present paper, we have substantially relaxed the restrictions on $r_{ \pm}$, however, at the cost of $C^{1}$-regularity of solutions. Our study of Lipschitz isometric maps $f:\left(M, g_{1}, g_{2}\right) \rightarrow\left(\mathbb{R}^{q}, h_{1}, h_{2}\right)$ relies extensively on the convex integration theory which incorporates the essence of the approach of Kuiper [1955]. The key idea of the method of convex integration can be stated as follows: If $A$ is a connected subset of $\mathbb{R}^{q}$ such that the interior of the convex hull of $A$ contains the origin then there is a $C^{1}$-map $f: S^{1} \rightarrow \mathbb{R}^{q}$ whose derivative maps $S^{1}$ into $A$. This can be viewed as the convex integration over a circle. However, in this paper we obtain only Lipschitz solutions in contrast with $C^{1}$-solutions in 
[D'Ambra and Datta 2002]. The reason behind this is that we are unable to solve the connectivity problem for the subsets of the form $S_{1} \cap S_{2} \cap T$, where $S_{1}$ and $S_{2}$ are two spheres in $\mathbb{R}^{q}$ relative to the metrics $h_{1}$ and $h_{2}$ respectively and $T$ is an affine subspace in $\mathbb{R}^{q}$.

We organize the paper as follows. We devote Section 2 to review the basic language of $h$-principle theory and convex integration techniques to deal with open first order partial differential relations. In Section 3 we introduce the notion of $\left(h_{1}, h_{2}\right)$-regularity for $C^{1}$-maps $f: M \rightarrow \mathbb{R}^{q}$ and study the geometry underlying the regularity condition which plays a crucial role in our treatment. In Section 4 we prove the Main Lemma (Lemma 4.1) leading to Theorem 1.1 and in Section 5 we prove the existence of an approximate solution to our problem. The proof of the Main Theorem (Theorem 1.1) is given in Section 6. The one-dimensional case is separately studied in Section 7 where we show that there exists, in fact, a $C^{1}$-solution.

\section{Review of convex integration techniques}

In this section we recall the terminology of the theory of $h$-principle and discuss in brief the main result of convex integration technique following [Eliashberg and Mishachev 2002].

Let $f$ be the germ of some local $C^{r}$-map at $x \in M$. The $r$-jet of $f$ at $x$ is by definition the ordered tuple

$$
j_{f}^{r}(x)=\left(x, f(x), D f(x), \ldots, D^{r} f(x)\right),
$$

where $D^{k} f$ denotes the derivative map of $f$ of order $k$. The collection of all such $r$-jets constitutes the total space of a fibre bundle over $M$ which is denoted by $p^{r}: J^{r}(M, N) \rightarrow M$. The bundle is referred as the $r$-jet bundle associated with the space of $C^{r}$-maps from $M$ to $N$.

If $r=1$ then

$$
j_{f}^{1}(x)=(x, f(x), D f(x))
$$

and $J^{1}(M, N)$ can be identified with the total space of the bundle Hom $(T M, T N)$.

A continuous map $\sigma: M \rightarrow J^{1}(M, N)$ is said to be a section if $p^{r} \circ \sigma=\mathrm{id}_{M}$. If $f: M \rightarrow N$ is a $C^{r}$-map then its $r$-jet map $j_{f}^{r}$ defined by

$$
j_{f}^{r}(x)=\left(x, f(x), D f(x), \ldots, D^{r} f(x)\right)
$$

is a section of $p^{r}$.

Definition 2.1. An $r$-th order partial differential relation is a subset $\mathscr{R}$ of $J^{r}(M, N)$. A $C^{r}$-map $f: M \longrightarrow N$ is said to be a solution of $\mathscr{R}$ if its $r$-jet map $j_{f}^{r}$ maps $M$ into $\mathscr{R}$. 
A section of $p^{r}$ whose image is contained in $\mathscr{R}$ is called a formal solution of the differential relation. A formal solution of $\mathscr{R}$ is said to be holonomic if it is the $r$-jet map of some $C^{r}$-map $f: M \rightarrow N$.

A differential relation $\mathscr{R}$ is said to satisfy the $h$-principle if every formal solution $\sigma$ can be homotoped to a holonomic section in the space of all formal solutions.

Definition 2.2. Let $\Omega$ be an open subset of a manifold $M$. A continuous map $f$ from $\Omega$ into a manifold $N$ is said to be piecewise $C^{r}$ if there exists a countable system of mutually disjoint open sets $\Omega_{j} \subset \Omega$ which cover $\Omega$ up to a set of measure zero and the restriction of $f$ to each $\Omega_{j}$ is $C^{r}$.

Let $\mathscr{R} \subset J^{r}(M, N)$ be an $r$-th order differential relation. A piecewise $C^{r}$-map $f: M \rightarrow N$ is said to be a piecewise $C^{r}$-solution of $\mathscr{R}$ if $j_{f}^{r}(x) \in \mathscr{R}$ for all $x \in M$ where the $r$-th derivative of $f$ exists.

The convex integration technique gives solutions to $h$-principle for some differential relations which satisfy certain convexity condition. The key idea of the convex integration technique is stated in the following lemma.

Lemma 2.3 [Gromov 1986, 2.4.1]. Let $A$ be a connected subset of $\mathbb{R}^{q}$ and let $\mathbf{0}$ belong to the interior of the convex hull of $A$. Then there exists a $C^{1}$-map $f$ : $[0,1] \rightarrow \mathbb{R}^{q}$ such that $f^{\prime}(t) \in A$ for all $t \in[0,1]$. Moreover, $f$ can be made to lie in an arbitrary small neighbourhood of $\mathbf{0}$.

If the connectivity condition on $A$ is dropped in the above lemma then it delivers a piecewise linear map $f$ such that $f(0)=f(1)=\mathbf{0}$ and $f^{\prime}(t) \in A$ whenever $f$ is differentiable [Eliashberg and Mishachev 2002, §17.4(D)]. More generally we obtain:

Proposition 2.4. Let $\mathscr{R}$ be an open subset of $J^{1}\left(\mathbb{R}, \mathbb{R}^{q}\right)$ and let $f:[0,1] \rightarrow \mathbb{R}^{q}$ be a continuous function which is $C^{1}$ on $(0,1)$. Suppose that $j_{f}^{1}(x)$ lies in the convex hull of $\mathscr{R}_{b(x)}$ for all $x \in(0,1)$, where

$$
b(x)=(x, f(x)) \in J^{0}\left(\mathbb{R}, \mathbb{R}^{q}\right) .
$$

Then $f$ can be homotoped to a piecewise $C^{1}$-solution $f_{1}$ of $\mathscr{R}$ in any $C^{0}$-neighbourhood of $f$ such that $f_{1}(0)=f(0)$ and $f_{1}(1)=f(1)$.

Proof. Consider any $\varepsilon>0$. Appealing to one-dimensional convex integration [Eliashberg and Mishachev 2002, §17.3] we can construct a piecewise linear map $f^{1}$ on the interval $[\varepsilon, 1-\varepsilon]$ which coincides with $f$ at the boundary points and is a piecewise $C^{1}$-solution of $\mathscr{R}$ on $(\varepsilon, 1-\varepsilon)$. Next consider, for each $n \geq 1$, a pair of disjoint intervals $I_{n}=\left[\varepsilon / 2^{n}, \varepsilon / 2^{n-1}\right]$ and $J_{n}=\left[1-\varepsilon / 2^{n-1}, 1-\varepsilon / 2^{n}\right]$. The interior of these sets cover $[0,1]$ up to a set of measure zero. Now, applying [Eliashberg and Mishachev 2002, §17.3] again to the restriction of $f$ to $I_{n} \cup J_{n}$ we obtain a piecewise linear map $f^{n}$ on $I_{n} \cup J_{n}$ which coincides with $f$ at the endpoints and 
satisfies the differential relation except at the points where the derivative does not exist. Further, we can choose $f^{n}$ to be $\varepsilon / 2^{n}$-close to $f$ on the set. Now all these maps patch together to give a piecewise linear map $f_{1}$ on $(0,1)$. Further, this map extends continuously to the closed interval $[0,1]$ and the extended map satisfies the desired conditions.

Remark. If $f$ is a solution of $\mathscr{R}$ on a neighbourhood of some closed subset $K$, then the homotopy remains constant on some (possibly smaller) open neighbourhood of $K$.

The result above may be generalised to a parametric version following [Eliashberg and Mishachev 2002, §17.5.1].

Proposition 2.5. Let $\mathscr{R}$ be an open subset of $I^{l} \times J^{1}\left(\mathbb{R}, \mathbb{R}^{q}\right)$ and

$$
\mathscr{R}_{p}=p \times J^{1}\left(\mathbb{R}, \mathbb{R}^{q}\right) \cap \mathscr{R} .
$$

Let $f: I^{l} \times I \rightarrow \mathbb{R}^{q}$ be a continuous function which is $C^{1}$ in the interior of $I^{l} \times I$. Let $f_{p}$ denote the restriction of $f$ to $p \times I$ and suppose that for each $p$, the pair $\left(f_{p}, \mathscr{R}_{p}\right)$ satisfies the hypothesis of Proposition 2.4. Then $f$ can be homotoped to a piecewise $C^{1}$-map $f_{1}$ in any $C^{0}$-neighbourhood of $f$ such that

(1) $\left(f_{1}\right)_{p}$ is a piecewise $C^{1}$-solution of $\mathscr{R}_{p}$;

(2) $f_{1}=f$ on $I^{l} \times\{0,1\}$;

(3) the first order derivatives of $f_{1}(p, t)$ with respect to $p$ are arbitrarily $C^{0}$ close to the respective derivatives of $f(p, t)$.

Further, if $f_{p}$ is a genuine solution of $\mathscr{R}_{p}$ for $p \in \mathcal{O} p \partial I^{l}$ then the homotopy can be kept constant for $p \in \mathcal{O} p \partial I^{l}$. (The notation $\mathcal{O} p \partial I^{l}$ is used to denote a nonspecified open neighbourhood of $\partial I^{l}$ which may become smaller in the course of the argument.)

We shall now state the main result on convex integration which yields piecewise $C^{1}$-solutions to certain open relations. Before stating it we need to recall the basic language of $\perp$-jets.

Let $\tau$ be an integrable hyperplane field on $\mathbb{R}^{n}$. With respect to this $\tau$ we define an equivalence relation $\sim$ on $J^{1}\left(\mathbb{R}^{n}, \mathbb{R}^{q}\right)$ as follows: If $(x, y, \alpha),(x, y, \beta)$ lie in the same fibre over $(x, y) \in J^{0}\left(\mathbb{R}^{n}, \mathbb{R}^{q}\right)$, then

$$
\alpha \sim \beta \quad \text { if and only if }\left.\quad \alpha\right|_{\tau}=\left.\beta\right|_{\tau} .
$$

The equivalence class of $(x, y, \alpha)$, denoted as $P_{\alpha}$, is an affine subspace of dimension $q$ in the jet space. Indeed, if we fix a vector field $\mathbf{v}$ on $\mathbb{R}^{n}$ transversal to $\tau$, then $(x, y, \beta) \in P_{\alpha}$ is completely determined by $\beta(\mathbf{v}) \in \mathbb{R}^{q}$. Thus relative to $\tau$ we can slice the 1 -jet space into $q$-dimensional affine subspaces $P_{\alpha} . P_{\alpha}$ is 
called the principal subspace through $(x, y, \alpha)$ corresponding to $\tau$. The set of equivalence classes is denoted by $J^{\perp}\left(\mathbb{R}^{n}, \mathbb{R}^{q}\right)$ and there is a canonical projection $p: J^{1}\left(\mathbb{R}^{n}, \mathbb{R}^{q}\right) \rightarrow J^{\perp}\left(\mathbb{R}^{n}, \mathbb{R}^{q}\right)$ which takes a 1 -jet onto its equivalence class. Let $j_{f}^{\perp}=p \circ j_{f}^{1}$.

Identifying $j_{f}^{1}(x)$ with $\left(j_{f}^{\perp}(x), d f_{x}(\mathbf{v})\right)$ we can write

$$
J^{1}\left(\mathbb{R}^{n}, \mathbb{R}^{q}\right)=J^{\perp}\left(\mathbb{R}^{n}, \mathbb{R}^{q}\right) \times \mathbb{R}^{q} .
$$

Note that when $n=1, J^{\perp}\left(\mathbb{R}^{1}, \mathbb{R}^{q}\right)=J^{0}\left(\mathbb{R}^{1}, \mathbb{R}^{q}\right)=\mathbb{R} \times \mathbb{R}^{q}$.

Theorem 2.6. Let $\mathscr{R}$ be an open subset of $J^{1}\left(\mathbb{R}^{n}, \mathbb{R}^{q}\right)$. Let $f_{0}: I^{n} \rightarrow \mathbb{R}^{q}$ be a piecewise $C^{1}$-function such that $j_{f_{0}}^{1}(x)$ lies in the convex hull of $\mathscr{R}_{b(x)}$ whenever the derivative exists, where $b(x)=j \frac{\perp}{f_{0}}(x)$. Then there exists a piecewise $C^{1}$-solution of $\Re, f_{1}: I^{n} \rightarrow \mathbb{R}^{q}$, which is homotopic to $f_{0}$. Moreover, the homotopy can be made to lie in an arbitrary $C^{0}$-neighbourhood of $f_{0}$.

Further, if $f_{0}$ is a piecewise $C^{1}$-solution of $\mathscr{R}$ on some open neighbourhood of a compact set $K \subset I^{n}$, then the homotopy remains constant on some (possibly smaller) neighbourhood of $K$.

For the sake of completeness we include the proof from [Eliashberg and Mishachev 2002].

Proof. Consider the splitting of the cube $I^{n}$ as $I^{n-1} \times I$. Form a relation

$$
\mathscr{R}^{1} \subset I^{n-1} \times J^{1}\left(\mathbb{R}, \mathbb{R}^{q}\right)
$$

fibred over $I^{n-1}$ as follows:

For each $x \in I^{n}$ let $P\left(j_{f}^{\perp}(x)\right)$ denote the principal subspace through $j_{f}^{1}(x)$ corresponding to the splitting $I^{n-1} \times I$. Let $\Omega(f(p, t))$ be the subset defined by

$$
\left\{j_{f}^{\perp}(p, t)\right\} \times \Omega(f(p, t))=P\left(j_{f}^{\perp}(p, t)\right) \cap \Re .
$$

By the given hypothesis, $\partial_{t} f(p, t)$ belongs to the convex hull of $\Omega(f(p, t))$ in $P\left(j_{f}^{\perp}(x)\right)$. Since $\mathscr{R}$ is open there is an open neighbourhood $D_{\varepsilon}^{q}(f(p, t))$ of $f(p, t)$ in $\mathbb{R}^{q}$ and an open subset $\Omega^{\prime}(f(p, t))$ contained in $\Omega(f(p, t))$ such that

(1) $\Omega^{\prime}(f(p, t))$ contains $\partial_{t} f(p, t)$ in its convex hull and

(2) $\{(p, t)\} \times D_{\varepsilon}^{q}(f(p, t)) \times\left\{\partial_{p} f(p, t)\right\} \times \Omega^{\prime}(f(p, t)) \subset \Re$ for all $(p, t) \in I^{n-1} \times I$.

In the above, $\partial_{t}$ and $\partial_{p}$ respectively denote the derivatives of the function with respect to the coordinates $t$ and $p$.

For each $p \in I^{n-1}$ define a relation $\mathscr{R}_{p}^{1} \subset J^{1}\left(\mathbb{R}, \mathbb{R}^{q}\right)$ as

$$
\mathscr{R}_{p}^{1}=\left\{(t, y, v) \in I \times \mathbb{R}^{q} \times \mathbb{R}^{q}: y \in D_{\varepsilon}^{q}(f(p, t)), v \in \Omega^{\prime}(f(p, t))\right\} .
$$

Then $\mathscr{R}^{1}=\bigcup_{p}\{p\} \times \mathscr{R}_{p}^{1}$ is a fibred relation in $I^{n-1} \times J^{1}\left(\mathbb{R}, \mathbb{R}^{q}\right)$ which is defined over an open neighbourhood of the graph of the section $f$ in $I^{n} \times \mathbb{R}^{q}$. 
Further for an appropriate choice of $\Omega^{\prime}(f(p, t))$ we may assume that $\mathscr{R}^{1}$ is an open fibred relation in $I^{n-1} \times J^{1}\left(\mathbb{R}, \mathbb{R}^{q}\right)$.

Also note that for a fixed $p \in I^{n-1}, t \mapsto f(p, t)$ is a short solution of $\mathscr{R}_{p}^{1}$.

We now apply the parametric one-dimensional convex integration to obtain a piecewise $C^{1}$-homotopy $f_{\tau}$ of fibrewise "short" (see [Eliashberg and Mishachev 2002] for the definition) solutions of $\mathscr{R}^{1}$ which is $C^{0}$ close to $f$ and satisfies

$$
f_{\tau}(p, 0)=f(p, 0) \quad \text { and } \quad f_{\tau}(p, 1)=f(p, 1)
$$

for all $p \in I^{n-1}$. Furthermore, the first order derivatives of $f_{1}(p, t)$ with respect to the parameter $p$ (wherever exist) are arbitrarily $C^{0}$ close to the respective derivatives of $f(p, t)$. Hence,

$$
\left(f_{1}(p, t), \partial_{p} f(p, t), \partial_{t} f_{1}(p, t)\right) \in \mathscr{R} .
$$

Since $\mathscr{R}$ is open and since the derivatives of $f_{1}$ with respect to $p$ are arbitrarily close to the respective derivatives of $f$ it follows that

$$
\left(f_{1}(p, t), \partial_{p} f_{1}(p, t), \partial_{t} f_{1}(p, t)\right) \in \mathscr{R} .
$$

Thus $f_{1}$ is a solution of $\mathscr{R}$ with the desired properties.

Remark 2.7. We refer the reader to [Gromov 1986, p. 218] for a general result on the existence of (almost everywhere differentiable) Lipschitz solutions to some differential relations.

\section{3. $\left(h_{1}, h_{2}\right)$ regularity and underlying geometry}

Throughout this section $h_{1}$ and $h_{2}$ will denote two positive definite symmetric bilinear forms on $\mathbb{R}^{q}$. For any subspace $V$ of $\mathbb{R}^{q}$, we shall denote its orthogonal complement with respect to $h_{i}$ by $V^{\perp_{i}}$ for $i=1,2$.

Definition 3.1. A subspace $V$ of $\mathbb{R}^{q}$ is said to be $\left(h_{1}, h_{2}\right)$-regular if $V^{\perp_{1}}$ is transversal to $V^{\perp_{2}}$.

Observe that if $A: \mathbb{R}^{q} \longrightarrow \mathbb{R}^{q}$ is the (unique) linear transformation defined by $h_{2}(v, w)=h_{1}(A v, w)$ for all $v, w \in \mathbb{R}^{q}$, then a subspace $V$ in $\mathbb{R}^{q}$ is regular if and only if $V+A(V)$ has the maximum dimension.

Definition 3.2. A vector $v \in \mathbb{R}^{q}$ is said to be $\left(h_{1}, h_{2}\right)$-regular provided the onedimensional subspace $\langle v\rangle$ spanned by $v$ is a $\left(h_{1}, h_{2}\right)$-regular subspace of $\mathbb{R}^{q}$.

Observation 1. A vector $v$ is $\left(h_{1}, h_{2}\right)$-regular if and only if $v$ and $A v$ are linearly independent, $A: \mathbb{R}^{q} \longrightarrow \mathbb{R}^{q}$ being the unique linear map defined above. Consequently, the set of nonregular vectors precisely consists of the eigen-vectors of A. 
The following observation brings out the underlying geometry of the $\left(h_{1}, h_{2}\right)$ regular vectors.

Observation 2. Let $\left(\mathbb{R}^{q}, h_{1}, h_{2}\right)$ be as in the above. We shall denote the norms of a vector $w \in \mathbb{R}^{q}$ relative to $h_{1}$ and $h_{2}$ by $\|w\|_{1}$ and $\|w\|_{2}$ respectively. Let

$$
S_{r}=\left\{w \in \mathbb{R}^{q} \mid\|w\|_{1}=r\right\} \quad \text { and } \quad E_{r}=\left\{w \in \mathbb{R}^{q} \mid\|w\|_{2}=r\right\}
$$

denote the spheres of radius $r$ in $\mathbb{R}^{q}$ relative to the two metrics. Observe that, a vector $v \in S_{r} \cap E_{r^{\prime}}$ is $\left(h_{1}, h_{2}\right)$-regular if and only if $S_{r}$ and $E_{r^{\prime}}$ intersect transversally at $v$. Indeed, $v$ is a regular vector if and only if $v^{\perp_{1}}$ is transversal to $v^{\perp_{2}}$. If $v \in S_{r} \cap E_{r^{\prime}}$, then $v^{\perp_{1}}$ is tangent to $S_{r}$ at $v$ and $v^{\perp_{2}}$ is tangent to $E_{r^{\prime}}$ at $v$. Therefore it follows that $S_{r}$ is transversal to $E_{r^{\prime}}$ at $v$.

Observation 3. Let $V$ be a $\left(h_{1}, h_{2}\right)$-regular subspace of $\mathbb{R}^{q}$ of dimension $(n-1)$ and let

$$
X=V^{\perp_{1}} \cap V^{\perp_{2}}=(V \oplus A(V))^{\perp_{1}} .
$$

Then $X$ has codimension $2(n-1)$ in $\mathbb{R}^{q}$. For any vector $w \in \mathbb{R}^{q}, \tau \oplus\langle w\rangle$ is an $\left(h_{1}, h_{2}\right)$-regular subspace if and only if $w^{\perp_{1}} \cap X$ is transversal to $w^{\perp_{2}} \cap X$ in $X$. Indeed, $V \oplus\langle w\rangle$ is a $\left(h_{1}, h_{2}\right)$-regular subspace if and only if $(V \oplus\langle w\rangle)^{\perp_{1}}$ is transversal to $(V \oplus\langle w\rangle)^{\perp_{2}}$, that is, if and only if

$$
\operatorname{codim}\left((V \oplus\langle w\rangle)^{\perp_{1}} \cap(V \oplus\langle w\rangle)^{\perp_{2}}\right)=2 n .
$$

This is equivalent to saying $X \cap w^{\perp_{1}} \cap w^{\perp_{1}}$ has codimension 2 in $X$. Thus $w^{\perp_{1}} \cap X$ is transversal to $w^{\perp_{2}} \cap X$.

Let $T$ be a translate of $X$ through $w$. Suppose that $r=\|w\|_{1}$ and $r^{\prime}=\|w\|_{2}$. Since $w^{\perp_{1}} \cap X$ is the tangent space of $S_{r} \cap T$ at $w$ and $w^{\perp_{2}} \cap X$ is the tangent space of $E_{r^{\prime}} \cap T$ at $w$, it follows from the above that the sets $S_{r} \cap T$ and $E_{r}^{\prime} \cap T$ intersect transversally in $T$ at $w$.

In particular, we can show that if $w$ is in $X$, then $V \oplus\langle w\rangle$ is $\left(h_{1}, h_{2}\right)$-regular if and only if $w$ is $\left(\bar{h}_{1}, \bar{h}_{2}\right)$-regular, where $\bar{h}_{1}$ and $\bar{h}_{2}$ denote the restrictions of $h_{1}$ and $h_{2}$ respectively to $X$.

Let $\bar{A}$ denote the unique linear transformation $X \rightarrow X$ such that

$$
\bar{h}_{2}(v, w)=\bar{h}_{1}(\bar{A} v, w) \text { for } \quad v, w \in V .
$$

If $w \in X$ is $\left(\bar{h}_{1}, \bar{h}_{2}\right)$-regular then $w$ and $\bar{A}(w)$ are linearly independent. Let $A(w)=$ $x+x^{\perp}$, where $x \in X$ and $x^{\perp} \in X^{\perp_{1}}$. Then

$$
h_{2}(w, v)=h_{1}(A w, v)=h_{1}\left(x+x^{\perp}, v\right)=h_{1}(x, v)
$$

for all $v \in X$. Hence $x=\bar{A}(w)$. This proves that $A w=\bar{A} w+x^{\perp}$. Since $w, \bar{A} w$ are linearly independent in $X$ and $x^{\perp} \notin X$ it follows that $w$ and $A w$ are linearly independent and consequently, $V \oplus\langle w\rangle$ is $\left(h_{1}, h_{2}\right)$-regular. 
Definition 3.3. Let $N$ be a smooth manifold with two Riemannian metrics $h_{1}$ and $h_{2}$. A smooth map $f: M \rightarrow N$ will be called $\left(h_{1}, h_{2}\right)$-regular if for each $x \in M$, $d f_{x}\left(T_{x} M\right)$ is a $\left(h_{1}, h_{2}\right)$-regular subspace of $T_{f(x)} N$.

Proposition 3.4 [D'Ambra and Datta 2002]. Let $h_{1}, h_{2}$ be two positive definite symmetric bilinear forms on $\mathbb{R}^{q}$ such that the eigen-values of $A$ (as defined above) are all distinct. Then a generic map $f: M \longrightarrow \mathbb{R}^{q}$ is $\left(h_{1}, h_{2}\right)$-regular if q exceeds $3 \operatorname{dim} M-1$.

\section{The Main Lemma}

Let $M$ be a smooth manifold of dimension $n$. Let $\mathbb{R}^{q}$ be the $q$-dimensional Euclidean space. In what follows $h_{1}$ and $h_{2}$ will denote two Euclidean metrics on $\mathbb{R}^{q}$ which satisfy the following conditions:

There exist two numbers $0<a<b$, such that

(1) $c^{2} h_{1}-h_{2}$ is a nondegenerate indefinite form for each real number $c$ lying in $[a, b]$;

(2) $r_{+}\left(a^{2} h_{1}-h_{2}\right) \geq 2 n$ and $r_{-}\left(b^{2} h_{1}-h_{2}\right) \geq 2 n$, where $r_{+}$and $r_{-}$denote respectively the positive and the negative ranks of an indefinite metric; and

(3) if $A: \mathbb{R}^{q} \rightarrow \mathbb{R}^{q}$ is the unique linear isomorphism given by $h_{2}(v, w)=$ $h_{1}(A v, w)$ for all $v, w \in \mathbb{R}^{q}$, then $A$ has distinct eigenvalues.

Lemma 4.1. Let $g_{1}$ and $g_{2}$ be two Riemannian metrics on $M$ which are related by $a^{2} g_{1}<g_{2}<b^{2} g_{2}$. Let $f: M \rightarrow \mathbb{R}^{q}$ be an $\left(h_{1}, h_{2}\right)$-regular immersion such that

$$
g_{1}-f^{*} h_{1}=\phi^{2} d \psi^{2} \quad \text { and } \quad g_{2}-f^{*} h_{2}=c^{2} \phi^{2} d \psi^{2},
$$

where $\phi, \psi$ are smooth functions on $M, \phi$ has compact support contained in an open set $U$ of $M$ and $a<c<b$.

Then there exists a piecewise $C^{1}$-map $\bar{f}$ which is a fine $C^{0}$-approximation of $f$ and has the following properties:

(1) $\bar{f}$ coincides with $f$ outside $U$;

(2) $\bar{f}^{*} h_{i}$ is arbitrarily close to $g_{i}\left(\bar{f}^{*} h_{i} \approx g_{i}\right)$ for $i=1,2$ relative to the fine $C^{0}$-topology on each component where $\bar{f}$ is $C^{1}$.

Proof. Let $\Phi$ denote the subset of $J^{1}\left(M, \mathbb{R}^{q}\right)$ consisting of all 1-jets $(x, y, \alpha)$ such that $\alpha^{*} h_{1}=g_{1}$ and $\alpha^{*} h_{2}=g_{2}$. Let $\tau$ be the hyperplane field over $U$ defined by $\operatorname{ker} d \psi$. Then $\tau$ is integrable and its integral submanifolds are precisely the level sets of the function $\psi$.

Consider the bundle

$$
p_{\perp}^{1}: J^{(1)}\left(U, \mathbb{R}^{q}\right) \rightarrow J^{\perp}\left(U, \mathbb{R}^{q}\right)
$$


relative to the hyperplane distribution $\tau$ on $U$. An element $b$ of $J^{\perp}\left(M, \mathbb{R}^{q}\right)$ is of the form $b=(x, y, \beta)$, where $x \in U, y \in \mathbb{R}^{q}$ and $\beta: \tau_{x} \rightarrow \mathbb{R}^{q}$ is a linear map. The fibre over $b$ consists of all linear maps $\alpha: T_{x} M \rightarrow \mathbb{R}^{q}$ which restricts to $\beta$ on $\tau_{x}$.

To describe the intersection of the relation $\mathscr{I}$ with the principal subspaces of the fibration $p_{\perp}^{1}$, we choose a vector field $\mathbf{v}_{0}$ on $T U$ such that

$$
\left\|\mathbf{v}_{0}\right\|_{1}=\sqrt{g_{1}\left(\mathbf{v}_{0}, \mathbf{v}_{0}\right)}=1 \quad \text { and } \quad g_{1}\left(\mathbf{v}_{0}, \tau\right)=0
$$

on $U \supset \operatorname{supp} \phi$. Let $\left\|\mathbf{v}_{0}\right\|_{2}=\sqrt{g_{2}\left(\mathbf{v}_{0}, \mathbf{v}_{0}\right)}=r$; then $r$ is a smooth function on $U$ satisfying the inequality $a<r(x)<b$ for all $x \in U$. Let $p^{\prime}: \mathscr{I} \rightarrow J^{\perp}\left(M, \mathbb{R}^{q}\right)$ denote the restriction of $p_{\perp}^{1}$ to $\mathscr{\Im}$. Recall that a 1 -jet $(x, y, \alpha)$ in a principal subspace $J_{b}^{1}\left(U, \mathbb{R}^{q}\right)$ is completely determined by its value at $\mathbf{v}_{0}$. Moreover, if

$$
(x, y, \alpha) \in \Phi_{b}=J_{b}^{(1)}\left(U, \mathbb{R}^{q}\right) \cap \Im,
$$

then $\alpha\left(\mathbf{v}_{0}\right)$ is contained in the unique affine space

$$
T_{b}=\left\{w \in \mathbb{R}^{q} \mid h_{1}(w, \beta(\tau))=0 \text { and } h_{2}(w, \beta(v))=g_{2}\left(\mathbf{v}_{0}, v\right) \text { for all } v \in \tau\right\},
$$

where $b=(x, y, \beta) \in J^{\perp}\left(U, \mathbb{R}^{q}\right)$. Note that the equation $h_{2}(w, \beta(v))=g_{2}\left(v_{0}, v\right)$ defines an affine subspace of $\mathbb{R}^{q}$ which is a translate of $\beta(\tau)^{\perp_{2}}$. If $\alpha$ is $\left(h_{1}, h_{2}\right)$ regular then, in particular, $\beta\left(\tau_{x}\right)^{\perp_{1}}$ is transversal to $\beta\left(\tau_{x}\right)^{\perp_{2}}$ and the same is true for any translates of these spaces. Thus $T_{b}$ is an affine plane of codimension $2(n-1)$. Moreover, this is the translate of the vector subspace $X_{b}=\beta\left(\tau_{x}\right)^{\perp_{1}} \cap \beta\left(\tau_{x}\right)^{\perp_{2}}$ in $\mathbb{R}^{q}$.

Thus $J^{(1)}\left(U, \mathbb{R}^{q}\right) \cap \Im$ is contained in an affine subbundle of codimension 2(n-1) (over some open subset of $J^{\perp}\left(U, \mathbb{R}^{q}\right)$ ). Further, it follows that if

$$
\alpha \in J_{b}^{(1)}\left(U, \mathbb{R}^{q}\right) \cap \Phi
$$

then $\left\|\alpha\left(v_{0}\right)\right\|_{1}=1$ and $\left\|\alpha\left(v_{0}\right)\right\|_{2}=r$. Therefore we can characterize $J_{b}^{(1)}\left(U, \mathbb{R}^{q}\right) \cap \mathscr{}$ as

$$
J_{b}^{(1)}\left(U, \mathbb{R}^{q}\right) \cap \mathscr{I}=\left\{w \in T_{b}:\|w\|_{1}=1,\|w\|_{2}=r\right\} .
$$

We shall now show that the pair $(f, \mathscr{I})$ satisfies the conditions stated in the hypothesis of Theorem 2.6 except that $\mathscr{I}$ is not an open relation.

Notation. We fix the following notations for the subsequent discussion:

$$
S=\left\{w \in \mathbb{R}^{q}:\|w\|_{1}=1\right\}, \quad E=\left\{w \in \mathbb{R}^{q}:\|w\|_{2}=r\right\} .
$$

Sublemma 4.2. $j_{f}^{1}(x)$ lies in the convex hull of $\Phi_{b(x)}$ if $r_{ \pm}\left(c^{2} h_{1}-h_{2}\right) \geq 2 n$. In other words, $d f_{x}\left(\mathbf{v}_{0}\right)$ lies in the convex hull of the set

$$
\left\{w \in T_{b(x)}:\|w\|_{1}=1,\|w\|_{2}=r\right\},
$$

where $b(x)=j_{f}^{\perp}(x)$. 
Proof of Sublemma 4.2. Observe that

(1) $d f_{x}\left(\mathbf{v}_{0}\right)$ lies in $T_{b(x)}$, and

(2) $d f_{x}\left(\mathbf{v}_{0}\right)$ satisfies the equation

$$
c^{2}\left(1-\|w\|_{1}^{2}\right)=r^{2}-\|w\|_{2}^{2}
$$

since $g_{2}-f^{*} h_{2}=c^{2}\left(g_{1}-f^{*} h_{1}\right)$.

The above equation can be equivalently expressed as $\left(c^{2} h_{1}-h_{2}\right)(w, w)=c^{2}-r^{2}$. This represents a generalised hyperboloid $H$ since $r_{ \pm}\left(c^{2} h_{1}-h_{2}\right) \geq 2 n$. It may be seen easily that $H \cap S=E \cap S=H \cap E$.

Since $r_{ \pm}\left(c^{2} h_{1}-h_{2}\right) \geq 2 n, H$ is generated by affine subspaces of dimension $2 n-1$. To see this, let $h$ be a nondegenerate symmetric bilinear form on $\mathbb{R}^{q}$ of signature $\left(q_{+}, q_{-}\right)$. Let $v \in H$ be such that $h(v, v)=d \neq 0$ and let $V$ denote the $h$-orthogonal complement of the subspace generated by $v$. Then $V$ has dimension $n-1$ and

$$
r_{+}\left(\left.h\right|_{V}\right) \geq q_{+}-1, \quad r_{-}\left(\left.h\right|_{V}\right) \geq q_{-}-1 .
$$

Consequently, $V$ admits a regular $h$-isotropic subspace $I$ of dimension

$$
\min \left(q_{+}-1, q_{-}-1\right) .
$$

Here regularity means that $I$ does not intersect the kernel of $\left.h\right|_{V}$. Consider the affine subspace $W=I+v$. It is easy to see that $h(w, w)=d$ for every $w \in W$. This proves the above assertion.

Let $A_{x}$ be an affine subspace in $H$ which passes through $d f_{x}\left(\mathbf{v}_{0}\right)$. Since

$$
\operatorname{codim} T_{x}=2(n-1)<2 n-1=\operatorname{dim} A_{x},
$$

the intersection $T_{x} \cap A_{x}$ is an affine subspace of dimension at least 1. Since $d f_{x}\left(\mathbf{v}_{0}\right) \in T_{x} \cap A_{x}$ and $\left\|d f_{x}\left(\mathbf{v}_{0}\right)\right\|_{1}<1, T_{x} \cap A_{x} \cap S$ contains at least two points and $d f_{x}\left(\mathbf{v}_{0}\right)$ lies in the convex hull of this intersection. Noting that

$$
T_{x} \cap A_{x} \cap S \subset T_{x} \cap E \cap S,
$$

we conclude that $d f_{x}\left(\mathbf{v}_{0}\right)$ lies in the convex hull of $T_{x} \cap E \cap S$. This completes the proof of Sublemma 4.2.

Now we conclude the proof of the Main Lemma (Lemma 4.1). Since $\mathscr{I}$ is not an open relation we cannot directly apply Theorem 2.6 to the pair $(f, \mathscr{I})$. We take an arbitrary small open neighbourhood $\tilde{\Phi}$ of $\Phi$ and apply Theorem 2.6 to the pair $(f, \tilde{\Phi})$. Thus we obtain a fine $C^{0}$-approximation of $f$ by a piecewise $C^{1}$-solution $\bar{f}$ of $\tilde{\Phi}$. Choosing $\tilde{\Phi}$ sufficiently small, we can make $\bar{f}^{*} h_{1}$ and $\bar{f}^{*} h_{2}$ arbitrarily $C^{0}$ close to the pair $\left(g_{1}, g_{2}\right)$ as desired. This completes the proof. 


\section{Approximate solution}

We recall the definition of short maps from [D'Ambra and Datta 2002].

Definition 5.1. Let $M$ be a manifold with two Riemannian metrics $g_{1}$ and $g_{2}$. A $C^{1}$-map $f_{0}: M \longrightarrow\left(\mathbb{R}^{q}, h_{1}, h_{2}\right)$ is $\left(g_{1}, g_{2}\right)$-short if the metrics $g_{1}-f_{0}^{*}\left(h_{1}\right)$ and $g_{2}-f_{0}^{*}\left(h_{2}\right)$ on $M$ are positive definite. This will be expressed by $g_{i}-f_{0}^{*}\left(h_{i}\right)>0$ or $g_{i}>f_{0}^{*}\left(h_{i}\right)$, for $i=1,2$.

Proposition 5.2. Let $M$ be a $C^{\infty}$-manifold with two Riemannian metrics $g_{1}$ and $g_{2}$ which are related by $a^{2} g_{1}<g_{2}<b^{2} g_{1}$. Then there exists a $\left(g_{1}, g_{2}\right)$-short $C^{\infty}$ immersion $f_{0}: M \longrightarrow\left(\mathbb{R}^{q}, h_{1}, h_{2}\right)$ which also satisfies the inequalities

$$
\begin{aligned}
a^{2}\left(g_{1}-f_{0}^{*} h_{1}\right) & <\left(g_{2}-f_{0}^{*} h_{2}\right)<b^{2}\left(g_{1}-f_{0}^{*} h_{1}\right), \\
a^{2} f_{0}^{*} h_{1} & <f_{0}^{*} h_{2}<b^{2} f_{0}^{*} h_{1} .
\end{aligned}
$$

Proof. For any number $c$ with $a<c<b$, consider the nondegenerate form $\bar{h}=c^{2} h_{1}-h_{2}$. By the hypothesis of Theorem $1.1, r_{+}(\bar{h}) \geq 2 n$ and $r_{-}(\bar{h}) \geq 2 n$. Therefore, there exists a $C^{1}$-immersion $f: M \rightarrow \mathbb{R}^{q}$ such that $f^{*}(\bar{h})=0$. (This follows from an exercise in [Gromov 1986, 2.4.9, Corollary $\left(2^{\prime}\right)$ ]). Such an $f$ clearly satisfies the relation $a^{2} f^{*} h_{1}<f^{*} h_{2}<b^{2} f^{*} h_{1}$. Moreover, without any loss of generality we may assume that the map $f$ satisfying the above inequality is smooth, because if that is not the case we replace $f$ by a $C^{\infty}$-immersion which is sufficiently $C^{1}$ close to $f$.

Now, if $M$ is a closed manifold, then starting with an $f$ as above we can obtain the required $f_{0}$ by scaling the map $f$ with a suitable scalar (see the corresponding result in [D'Ambra and Datta 2002]). To obtain such an $f_{0}$ in the case of open manifolds we have to employ the partition of unity techniques.

Let $\mathscr{F}$ denote the set of all piecewise $C^{1}$-maps $f: M \rightarrow \mathbb{R}^{q}$ which satisfy the following conditions at each point $x \in M$ where $f$ is differentiable:

F1. $f$ is $\left(h_{1}, h_{2}\right)$-regular;

F2. $f$ is $\left(g_{1}, g_{2}\right)$-short;

F3. $a^{2}\left(g_{1}-f^{*} h_{1}\right)<g_{2}-f^{*} h_{2}<b^{2}\left(g_{1}-f^{*} h_{1}\right)$;

F4. $a^{2} f^{*} h_{1}<f^{*} h_{2}<b^{2} f^{*} h_{1}$.

Proposition 5.3. Let $f_{0}: M \rightarrow \mathbb{R}^{q}$ belong to $\mathscr{F}$ and let $0<\varepsilon<1$ be any positive number. Then there exists a piecewise $C^{1}$-map $f_{1} \in \mathscr{F}$ such that the following conditions are satisfied:

(1) $\varepsilon g_{1}<f_{1}^{*} h_{1}<g_{1}$ on the set of points where $f$ is differentiable;

(2) $f_{1}$ is arbitrarily close to $f_{0}$ in the fine $C^{0}$-topology. 
Remark 5.4. Condition (1) in the above proposition implies that $f_{1}$ is strictly $g_{1}$ short and the induced metric $f_{1}^{*} h_{1}$ is sufficiently close to $g_{1}$ when $\varepsilon$ is close to 1 .

Proof. Fix a locally finite open covering $\left\{U_{i}\right\}$ of $M$ by coordinate neighbourhoods. Since the metrics $g_{1}-f^{*} h_{1}$ and $g_{2}-f^{*} h_{2}$ are related by the inequalities (5-1) we can get simultaneous decomposition of $g_{1}-f^{*} h_{1}$ and $g_{2}-f^{*} h_{2}$ as

$$
\varepsilon\left(g_{1}-f^{*} h_{1}\right)=\sum_{i} \phi_{i}^{2} d \psi_{i}^{2} \text { and } \varepsilon\left(g_{2}-f^{*} h_{2}\right)=\sum_{i} c_{i}^{2} \phi_{i}^{2} d \psi_{i}^{2}
$$

where $c_{i}$ 's are constants which lie between $a$ and $b$, and $\phi_{i}$ 's and $\psi_{i}$ 's are smooth real valued functions. Further, for each $i$, the function $\phi_{i}$ has compact support contained in $U_{i}$ [D'Ambra and Datta 2002, Decomposition Lemma]. Let us define two sequences of Riemannian metrics $\left\{g_{1}^{i}\right\}$ and $\left\{g_{2}^{i}\right\}$ as

$$
g_{1}^{i}=g_{1}^{i-1}+\phi_{i}^{2} d \psi_{i}^{2} \text { and } g_{2}^{i}=g_{2}^{i-1}+c_{i}^{2} \phi_{i}^{2} d \psi_{i}^{2},
$$

where $g_{1}^{0}=f^{*} h_{1}$ and $g_{2}^{0}=f^{*} h_{2}$. Clearly, $g_{1}^{i}<g_{1}$ and $g_{2}^{i}<g_{2}$ for each $i$. Further, since $a^{2} f^{*} h_{1}<f^{*} h_{2}<b^{2} f^{*} h_{1}$ and $a<c_{i}<b$ for each $i, a^{2} g_{1}^{i}<g_{2}^{i}<b^{2} g_{1}^{i}$ for each $i$.

By applying the Main Lemma (Lemma 4.1) successively (with an appropriate choice of $\tilde{\Phi}$ for each $i$ ) we obtain a sequence of piecewise $C^{1}$-maps such that

$$
\bar{f}_{i}^{*} h_{\alpha} \approx g_{\alpha}^{i}
$$

for $\alpha=1,2, i=1,2, \ldots$ and $\bar{f}_{i}$ lies in a given neighbourhood of $f$ in the fine $C^{0}$-topology. Note that each $\bar{f}_{i}$ satisfies conditions F2 and F4. Since supp $\phi_{i} \subset U_{i}$ for each $i$, where $\left\{U_{i}\right\}$ is a locally finite open covering of $M$, the sequence $\bar{f}_{i}$ is eventually constant near any point $x \in M$. Therefore the sequence converges to a piecewise $C^{1}$-map on $V$. Let

$$
f_{1}=\lim _{i \rightarrow \infty} \bar{f}_{i}
$$

If $\bar{f}_{i}^{*} h_{\alpha}$ are sufficiently close to $g_{\alpha}^{i}$ for $\alpha=1,2$ and for all $i$, then $f_{1}$ can be made to satisfy F2, F3 and F4. Further,

$$
g_{1}-f_{1}^{*} h_{1} \approx g_{1}-\left(f^{*} h_{1}+\varepsilon\left(g_{1}-f^{*} h_{1}\right)\right)=(1-\varepsilon)\left(g_{1}-f^{*} h_{1}\right)<(1-\varepsilon) g_{1} .
$$

Hence $f_{1}$ satisfies $\varepsilon g_{1}<f_{1}^{*} h_{1}<g_{1}$.

\section{Proof of the Main Theorem}

We begin this section with some preliminaries on Lipschitz maps.

Definition 6.1. Let $(X, d)$ and $\left(Y, d^{\prime}\right)$ be two metric spaces and let $f: X \rightarrow Y$ be a continuous map. The map $f$ is said to be Lipschitz if there is a constant $K>0$ 
such that $d^{\prime}\left(f(x), f\left(x^{\prime}\right)\right)<K d\left(x, x^{\prime}\right)$ for all $x, x^{\prime} \in X . K$ is called the Lipscitz constant for $f$.

A Riemannian metric $g$ on a $C^{\infty}$-manifold $M$ induces a canonical metric space structure on $M$. If we denote this metric by $d_{g}$, then the distance $d_{g}\left(x, x^{\prime}\right)$ between two points $x, x^{\prime} \in M$ is defined to be the infimum of the lengths of all piecewise $C^{1}$-paths in $M$ joining $x$ and $x^{\prime}$.

Definition 6.2. A continuous map $f:(M, g) \rightarrow(N, h)$ from a Riemannian manifold $(M, g)$ into another Riemannian manifold $(N, h)$ will be called Lipschitz if it is a Lipschitz map relative to the metrics $d_{g}$ and $d_{h}$ on $M$ and $N$ respectively.

Example 6.3. A $C^{1}$-isometric map $f:(M, g) \rightarrow(N, h)$ between Riemannian manifolds is a Lipschitz map with a Lipschitz constant equal to 1. Hence, every $g$-short map is also a Lipschitz map.

A Riemannian metric $g$ on a manifold $M$ induces a canonical volume measure which we denote by $\mu_{g}$. Measurability on $(M, g)$ is therefore to be understood in terms of this $\mu_{g}$. Observe that if $g^{\prime}$ is another Riemannian metric on $M$ then a set $A$ in $M$ has measure zero relative to $\mu_{g}$ if and only if it has measure zero relative to $\mu_{g^{\prime}}$.

We recall the following facts about Lipschitz maps between Riemannian manifolds from [Weaver 1999].

- Every Lipschitz map between Riemannian manifolds is almost everywhere differentiable, since a Lipschitz map $f: \Omega \rightarrow \mathbb{R}^{q}$ defined on some open subset of $\mathbb{R}^{n}$ is almost everywhere differentiable.

- The Lipschitz functions on a Riemannian manifold are precisely those which have bounded measurable exterior derivative $d f$.

Definition 6.4. A Lipschitz map $f:(M, g) \rightarrow(N, h)$ from a Riemannian manifold $(M, g)$ into another Riemannian manifold $(N, h)$ will be called Lipschitz isometric if $d f_{x}: T_{x} M \rightarrow T_{f(x)} N$ is isometric for almost all $x \in M$.

- If $g_{1}$ and $g_{2}$ are two Riemannian metrics on a manifold $M$ satisfying $a^{2} g_{1}<$ $g_{2}<b^{2} g_{1}$ then a map $f: M \rightarrow \mathbb{R}^{q}$ is Lipschitz with respect to the pair $\left(g_{1}, h_{1}\right)$ if and only if it is Lipschitz with respect to the pair $\left(g_{2}, h_{2}\right)$, where $h_{1}, h_{2}$ are two linear metrics on $\mathbb{R}^{q}$. Therefore, there is no ambiguity when we speak of almost everywhere differentiable Lipschitz maps in the context of Theorem 1.1 .

Proof of Theorem 1.1. Since $\left(h_{1}, h_{2}\right)$-regular immersions are generic for $q \geq$ $3 \operatorname{dim} M$, it follows from Proposition 5.2 that there is a $\left(h_{1}, h_{2}\right)$-regular immersion $f_{0}: M \rightarrow \mathbb{R}^{q}$ which satisfies the inequalities in (5-1).

Let $\mathscr{R}$ denote the set of all 1 -jets $(x, y, \alpha)$ which satisfy the following properties: 
(1) $\alpha$ is short relative to both $\left(g_{1}, h_{1}\right)$ and $\left(g_{2}, h_{2}\right)$;

(2) $a^{2}\left(g_{1}-\alpha^{*} h_{1}\right)<g_{2}-\alpha^{*} h_{2}<b^{2}\left(g_{1}-\alpha^{*} h_{1}\right)$;

(3) $a^{2} \alpha^{*} h_{1}<\alpha^{*} h_{2}<b^{2} \alpha^{*} h_{1}$.

For every $\eta>0$ define relations $\mathscr{R}_{\eta}$ by

$$
\mathscr{R}_{\eta}=\mathscr{R} \cap\left\{(x, y, \alpha):(1-\eta) g_{1}<\alpha^{*} h_{1}<g_{1}\right\} .
$$

Let $\mathscr{I}$ denote the isometry relation

$$
\Phi=\left\{(x, y, \alpha) \in J^{1}\left(M, \mathbb{R}^{q}\right): \alpha^{*} h_{1}=g_{1}, \alpha^{*} h_{2}=g_{2}\right\},
$$

then:

- Each $\mathscr{R}_{\eta}$ is an open relation.

- The fibres of $\mathscr{I}$ over $J^{0}\left(M, \mathbb{R}^{q}\right)$ are compact sets. Hence, the relations $\mathscr{R}_{\eta}$ are uniformly bounded over compact sets in $M$.

- Let $\eta_{i}$ be a sequence of positive numbers such that $\eta_{i} \rightarrow 0$. If $\alpha_{i} \in \mathscr{R}_{\eta_{i}}$ and $\alpha_{i} \rightarrow \alpha$, then $\alpha \in \mathscr{I}$. (Compare with [Gromov 1986, p. 218].)

Let $\eta_{i}$ be a sequence of constants converging to zero and $\delta_{i}$ be a sequence of positive continuous functions on $M$ such that the series $\sum_{i} \delta_{i}$ converges pointwise on $M$. By applying Proposition 5.3 we obtain a sequence of piecewise $C^{1}$-maps $f_{i}: M \rightarrow \mathbb{R}^{q}$ for $i=1,2, \ldots$ such that $f_{i}$ is a piecewise $C^{1}$-solution of the relation $\mathscr{R}_{\eta_{i}}$ and the distance between $f_{i}(x)$ and $f_{i+1}(x)$ is less than $\delta_{i}(x)$ for all $x \in M$. Thus the sequence $\left\{f_{i}\right\}$ converges (in the $C^{0}$ compact open topology) to a continuous function $f$ on $M$. Since $f_{i}$ is a piecewise $C^{1}$-solution of the relation $\mathscr{R}_{\eta_{i}}$, it is Lipschitz (relative to $\left(g_{1}, h_{1}\right)$ ) and the Lipschitz constants of $f_{i}$ are uniformly bounded. Hence the limit function $f$ is also a Lipschitz map [Weaver 1999]. Consequently, $f$ is almost everywhere differentiable and the $L^{\infty}$ norm of $d f$ is finite on any coordinate neighbourhood of $M$.

We would further like to show that the sequence $d f_{i}, i=1,2, \ldots$, converges to $d f$ in $L^{1}(\Omega)$ for any compact coordinate neighbourhood $\Omega$. Since $L^{1}$ convergence of a sequence of functions guarantees the almost everywhere convergence of a subsequence of the original sequence to $d f$, this would imply that $f$ is a Lipschitz solution of $\mathscr{I}$ on all of $M$ (by a property of $\mathscr{R}_{\eta}$ discussed above).

However, to prove the desired $L^{1}$ convergence we need to choose the functions $\delta_{i}$ appropriately. First we fix a locally finite open covering of $M$ by coordinate neighbourhoods $\left\{\Omega_{\alpha}: \alpha=1,2, \ldots\right\}$. For our convenience we choose each $\Omega_{\alpha}$ to be compact. Suppose we have already constructed $\delta_{i}$ and $f_{i}$ for $i=1,2, \ldots, k$. Let $\left\{\varepsilon_{\alpha}\right\}$ be a sequence of positive numbers with $0<\varepsilon_{\alpha}<2^{-\alpha}$ such that

$$
\left\|d f_{i} * \rho_{\varepsilon_{\alpha}}-d f_{i}\right\|_{L^{1}\left(\Omega_{\alpha}\right)} \leq 2^{-\alpha} .
$$


The functions $\rho_{\varepsilon}$ are defined as in [Müller and Šverák 2003] by $\rho_{\varepsilon}=\varepsilon^{-n} \rho(x / \varepsilon)$, where $\rho: \mathbb{R}^{n} \rightarrow \mathbb{R}$ is the mollifying kernel, that is, a smooth nonnegative function supported in the open unit disc in $\mathbb{R}^{n}$ with $\int \rho d x=1$.

Observing that there exists a positive continuous function $\varepsilon$ on $M$ which is strictly less than $\varepsilon_{\alpha}$ on $\Omega_{\alpha}$ for each $\alpha=1,2, \ldots$, define

$$
\delta_{i+1}=\varepsilon \delta_{i} .
$$

Now we apply Proposition 5.3 to obtain a piecewise $C^{1}$-solution of $\mathscr{R}_{\eta_{i+1}}$ such that $\left|f_{i+1}-f_{i}\right|<\delta_{i+1}$. Proceeding this way we construct a sequence $\left\{f_{i}\right\}, i=1,2, \ldots$, which has all the desired property.

Now, arguing exactly as in [Müller and Šverák 2003, Theorem 3.2] we can prove that $d f_{i}$ converges to the derivative map of $f$ in $L^{1}\left(\Omega_{\alpha}\right)$ for each $\alpha$. This completes the proof of the theorem.

Remark 6.5. The proof of the main theorem begins with an immersion $f_{0}: M \rightarrow \mathbb{R}^{q}$ satisfying the inequalities (5-1). If $\mathbb{R}^{q}$ is replaced by a general manifold $N$ then such maps are no longer guaranteed. This is the main obstruction to generalise the result for arbitrary manifold $N$ in the place of $\mathbb{R}^{q}$. However, assuming the existence of such maps we may possibly prove the existence of Lipschitz isometric maps for pairs of Riemannian metrics [Gromov 1986, 2.4.9 (A)].

\section{One-dimensional case}

In this section we discuss the one-dimensional case which is the motivation to the general problem.

Let $M=S^{1}$ be the unit circle and let $g_{1}=d \theta^{2}$ be the canonical metric on $S^{1}$. Let $g_{2}=c^{2} g_{1}$. If $f: S^{1} \rightarrow \mathbb{R}^{q}$ is a $C^{1}$-immersion such that $f^{*} h_{i}=g_{i}$ for $i=1,2$ then

$$
\left\|\frac{\partial f}{\partial \theta}\right\|_{1}=1 \text { and }\left\|\frac{\partial f}{\partial \theta}\right\|_{2}=c,
$$

where $\|\cdot\|_{i}$ denote the norms relative to the metric $h_{i}$ for $i=1,2$. In other words, $\frac{\partial f}{\partial \theta} \in A$, where $A$ is given by

$$
A=\left\{\mathbf{y}=\left(y_{1}, \ldots, y_{q}\right) \in \mathbb{R}^{q}: \sum y_{i}^{2}=1 \text { and } \sum \lambda_{i}^{2} y_{i}^{2}=c^{2}\right\} .
$$

Lemma 7.1. Let $h_{1}$ and $h_{2}$ be two inner products on $\mathbb{R}^{q}$ such that $h_{1}-h_{2}$ is nondegenerate. Let $S_{1}$ and $S_{2}$ denote the unit spheres relative to the metrics $h_{1}$ and $h_{2}$ respectively. Then $S_{1} \cap S_{2}$ has the same homotopy type as $S^{r_{+}-1} \times S^{r_{-}-1}$, where $r_{+}$and $r_{-}$are respectively the positive and the negative ranks of $h_{1}-h_{2}$. Consequently, if $r_{ \pm} \geq 2$ then $S_{1} \cap S_{2}$ is connected. Further the interior of the convex hull of $S_{1} \cap S_{2}$ contains the origin. 
Proof. Let $h_{1}-h_{2}$ be nondegenerate. Note that a nonzero vector $v$ satisfies

$$
\left(h_{1}-h_{2}\right)(v, v)=0
$$

if and only if $\lambda v$ satisfies the same equation for all $\lambda$. This means that the onedimensional subspace $\ell_{v}$ containing $v$ lies completely inside the solution space $C$ of $h_{1}-h_{2}=0$. In other words, the solution space of this equation in $\mathbb{R}^{q}$ is a cone. Now, if $h$ is an arbitrary positive definite quadratic form on $\mathbb{R}^{q}$ then $\ell_{v}$ intersects the unit sphere relative to $h$ in exactly two points. Thus we see that $S_{1} \cap S_{2}$ has the same homotopy type as the space of nonzero solutions of the equation $h_{1}-h_{2}=0$. Choose basis vectors in $\mathbb{R}^{q}$ so that both $h_{1}$ and $h_{2}$ are in the diagonal form. The set $S_{1} \cap S_{2}$ has the same homeomorphism type as the solution space of the system of equations

$$
\begin{aligned}
& x_{1}^{2}+\cdots+x_{r_{+}}^{2}+y_{1}^{2}+\cdots+y_{r_{-}}^{2}=1, \\
& x_{1}^{2}+\cdots+x_{r_{+}}^{2}-y_{1}^{2}-\cdots-y_{r_{-}}^{2}=0,
\end{aligned}
$$

which is further equivalent to

$$
\begin{aligned}
& x_{1}^{2}+x_{2}^{2}+\cdots+x_{r_{+}}^{2}=\frac{1}{2}, \\
& y_{1}^{2}+y_{2}^{2}+\cdots+y_{r_{-}}^{2}=\frac{1}{2} .
\end{aligned}
$$

Therefore, $S_{1} \cap S_{2}$ has the homeomorphism type of $S^{r_{+}-1} \times S^{r_{-}-1}$, which is $k$ connected for $k \leq \min \left(r_{+}-2, r_{-}-2\right)$. Thus if $r_{ \pm} \geq 2$ then $S_{1} \cap S_{2}$ is connected and nowhere flat. (Note that in the lowest admissible dimension the intersection is topologically equivalent to a torus embedded in $S^{3}$.) Also note that if $\left(\bar{x}_{1}, \ldots, \bar{x}_{r_{+}}, \bar{y}_{1}, \ldots, \bar{y}_{r_{-}}\right) \in S_{1} \cap S_{2}$ then $\left( \pm \bar{x}_{1}, \ldots, \pm \bar{x}_{r_{+}}, \pm \bar{y}_{1}, \ldots, \pm \bar{y}_{r_{-}}\right) \in$ $S_{1} \cap S_{2}$, so that the convex hull of $S_{1} \cap S_{2}$ has nonempty interior and 0 belongs to the interior convex hull of $S_{1} \cap S_{2}$.

It follows from the above lemma that if $r_{ \pm}\left(c^{2} h_{1}-h_{2}\right) \geq 2$, then $A$ is connected and the interior of the convex hull of $A$ contains the origin. Thus, by Lemma 2.3 there exists a $C^{1}$-immersion $f: S^{1} \rightarrow \mathbb{R}^{q}$ such that $f^{*} h_{i}=g_{i}$ for $i=1,2$ when $r_{ \pm}\left(c^{2} h_{1}-h_{2}\right) \geq 2$.

On the other hand there does not exist any such isometric immersion if $q \leq 3$ since it is observed in [Gromov 1986, 2.4.1(A) Example] that if $f: S^{1} \rightarrow \mathbb{R}^{q}$ is a $C^{1}$-map whose derivative takes the unit circle $S^{1}$ into a (connected) subset $A$, then the convex hull of $A$ must contain the origin. Indeed, if $q=3$ and $h_{1}-h_{2}$ is a nondegenerate indefinite form, then $A$ is a disjoint union of two circles none of which contains the origin in its convex hull, thereby ruling out the existence of $C^{1}$-immersion with the desired isometry property.

We conclude the paper with a conjecture: 
Conjecture. If $r_{ \pm}\left(c^{2} h_{1}-h_{2}\right) \geq 2 n+1$ for all $c \in[a, b]$, then it is possible to obtain a $C^{1}$-solution of the general problem.

\section{Acknowledgements}

The authors would like to thank Misha Gromov for his valuable comments on this problem. The second author would also like to thank Vishwambhar Pati for sharing his ideas with her.

\section{References}

[D'Ambra and Datta 2002] G. D'Ambra and M. Datta, "Isometric $C^{1}$-immersions for pairs of Riemannian metrics", Asian J. Math. 6:2 (2002), 373-384. MR 2003f:53106 Zbl 1047.53030

[Eliashberg and Mishachev 2002] Y. Eliashberg and N. Mishachev, Introduction to the h-principle, Graduate Studies in Mathematics 48, Amer. Math. Soc., Providence, RI, 2002. MR 2003g:53164 Zbl 1008.58001

[Gromov 1986] M. Gromov, Partial differential relations, Ergebnisse der Mathematik und ihrer Grenzgebiete (3) 9, Springer, Berlin, 1986. MR 90a:58201 Zbl 0651.53001

[Kuiper 1955] N. H. Kuiper, "On $C^{1}$-isometric imbeddings. I, II", Nederl. Akad. Wetensch. Proc. Ser. A. 58 = Indag. Math. 17 (1955), 545-556, 683-689. MR 17,782c Zbl 0067.39601

[Müller and Šverák 2003] S. Müller and V. Šverák, "Convex integration for Lipschitz mappings and counterexamples to regularity", Ann. of Math. (2) 157:3 (2003), 715-742. MR 2005i:35028 Zbl 1083.35032

[Weaver 1999] N. Weaver, Lipschitz, algebras, World Sci., River Edge, NJ, 1999. MR 2002g:46002 Zbl 0936.46002

Received July 19, 2007. Revised February 29, 2008.

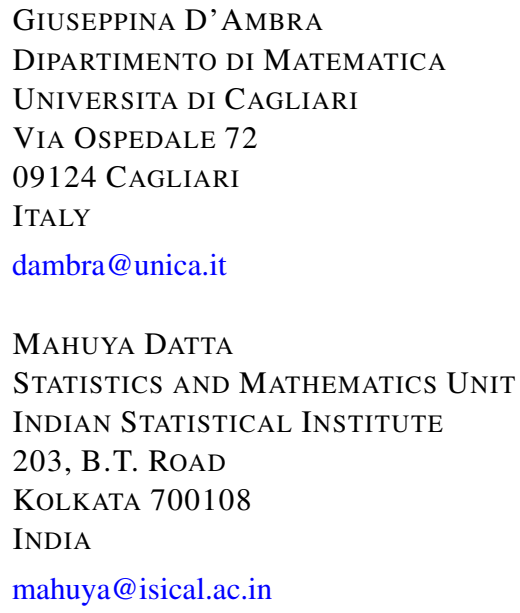

